\title{
Infrastructure-less Occupancy Detection and Semantic Localization in Smart Environments
}

\author{
Md Abdullah Al Hafiz Khan, H M Sajjad Hossain, Nirmalya Roy \\ Department of Information Systems \\ University of Maryland Baltimore County \\ mdkhan1@umbc.edu, riaj.sajjad@umbc.edu, nroy@umbc.edu
}

\begin{abstract}
Accurate estimation of localized occupancy related information in real time enables a broad range of intelligent smart environment applications. A large number of studies using heterogeneous sensor arrays reflect the myriad requirements of various emerging pervasive, ubiquitous and participatory sensing applications. In this paper, we introduce a zeroconfiguration and infrastructure-less smartphone based location specific occupancy estimation model. We opportunistically exploit smartphone's acoustic sensors in a conversing environment and motion sensors in absence of any conversational data. We demonstrate a novel speaker estimation algorithm based on unsupervised clustering of overlapped and non-overlapped conversational data and a change point detection algorithm for locomotive motion of the users to infer the occupancy. We augment our occupancy detection model with a fingerprinting based methodology using smartphone's magnetometer sensor to accurately assimilate location information of any gathering. We postulate a novel crowdsourcing-based approach to annotate the semantic location of the occupancy. We evaluate our algorithms in different contexts; conversational, silence and mixed in presence of 10 domestic users. Our experimental results on real-life data traces in natural settings show that using this hybrid approach, we can achieve approximately 0.76 error count distance for occupancy detection accuracy on average.
\end{abstract}

\section{Categories and Subject Descriptors}

H.4 [Mobile Systems and Applications]: Miscellaneous

\section{Keywords}

Crowdsourcing, Opportunistic sensing, Occupancy counting, Semantic Localization

\section{INTRODUCTION}

Localized commercial (University, Office, Mall, Cineplex, Restaurant etc.) and residential (apartment, home etc.) building occupancy detection and estimation at room/zone level granularity in real time can provide meaningful insights to many smart environment applications, such as green building, social gathering, event management etc. Smartphonebased participatory and citizen sensing applications have adhered to the promise of building such applications by utilizing various context sensing sensors on board. Different sensors can be exploited individually or in tandem to build a variety of such novel applications to satisfy the myriad requirements of differing smart environment applications. For example, potential benefit from microphone sensor based application is the assessment of social interaction and active engagement among a group of people by leveraging their conversational contents [1], speaker identification and characterization of social settings [2][3][4]. To enumerate the number of people in a conversational episode, such as during a social gathering, interactive lecture session or in a restaurant or shopping mall environment, various speaker counting paradigms have been explored [5][6][7]. Most of the recent studies which focus on conversational data features to extract high level occupancy information, assume that all of the users need to take turns at some point. While this specific scenario is feasible it is not ideal. To tackle this ideal situation, researchers have proposed using arrays of microphone sensors, video cameras or motion sensors for identifying microscopic occupancy information in real time [8][9] which are obtrusive in nature. We envision to move one step further by considering a more natural environment where people may spontaneously participate or abstain from any conversational phenomenon. We posit to augment smartphone-based locomotive sensing model in absence of any conversational episode along with acoustic sensing-based audio inference model to precisely capture the characteristic of a natural environment and accurately estimate the occupancy count. To further pinpoint the occupancy we integrate the smartphone's magnetometer sensor-based location sensing model. In pursuit of these goals we design a model which opportunistically exploits both the audio and motion data respectively from smartphone's microphone and accelerometer sensor to infer the number of people present in a gathering and their semantic location information as supplemented by the magnetometer sensor on the smartphone. We also introduce a crowdsourcing model to reduce the effort for obtaining semantic location information at scale.

In particular we propose a zero-hassle ambient and infrastructure-less mobile sensing (aka smartphone) based approach by exploiting only the smartphone's sensors to provide significantly greater visibility on real time occupancy 
and its semantic location. The key challenge in this case is to effectively estimate the number of people in a crowded and non-crowded environment either in presence of any conversational data or not. Such hybrid sensing approach could potentially furnish more fine-grained occupancy profiling to better serve many participatory sensing applications while saving smartphones' battery power by advocating a distributed sensing strategy. Main contributions of the paper are summarized below:

- We propose an acoustic sensing based linear time adaptive people counting algorithm based on real-life conversational data which promotes a unified strategy of considering both overlapped and non-overlapped conversational data in a natural environment. We propose to select opportunistically minimal number of microphone sensors which can substantially reduce the energy consumption of smartphones. Our proposed people counting algorithm can dynamically select length of the audio segment compared to the other existing work [6].

- Although acoustic sensing based approach holds great promises in inferring the number of occupants it fails in absence of any conversational data. Therefore we propose to augment motion sensing based counting strategy with our acoustic sensing based people counting algorithm which works on extreme modality of either of the data sources, be it acoustic or locomotive.

- We design a magnetometer sensor based localization technique at zone/room level granularity to infer the location of a conversing group. We propose a novel crowdsourcing model to map the magnetic signature of different locations and collect a large number of annotated location information to tag the occupancy with its semantic location information.

\section{RELATED WORK}

We particularly review the most relevant literatures on occupancy inference problem in the context of conversational sensing, localization, and speaker estimation which are smartphone based.

Smartphone Speaker Sensing: A large number of prior work have used smartphones' microphone to opportunistically analyze audio for context characterization. For example, SpeakerSense [4] performs speaker identification and SoundSense [10] classifies sounds from macro to micro contexts. They have often in common employing the supervised speaker learning techniques. In contrast, our model's occupancy counting process is entirely unsupervised. Our proposed model anonymously estimates the number of people from smartphones' acoustic cum locomotive sensing model where we have employed unsupervised learning techniques to cluster different forms of acoustic signatures. For example, [11] have built a model from mean and covariance matrices of Linear Predictive Cepstral Coefficient (LPCC) of voice segments in conversations and used Mahalanobis distance to determine if two models belong to the same or different speakers. [12] has performed speaker clustering using distance of the feature vectors extracted from different speakers and finally applied modified C-means algorithm with distance metric data. However, their experiments for occupants estimation were on telephonic conversational data, where multiple participants were present, and voices were frequently overlapped and intertwined with the noisy environment. Our proposed model performs speaker counting without any predefined environmental setup and collects data from natural conversation. Our proposed speaker counting algorithm is close to [13], [6] where smartphonebased speaker counting has been proposed in a controlled scenario where all the participants spoke actively. [6] used a fixed length audio segment $(3 \mathrm{sec})$ where each segment corresponds to an individual but we performed this audio segmentation dynamically to increase the accuracy of occupancy inference. [6] also classified a few segments as undetermined but our system never discards segments as undetermined which is achieved only through employing dynamic segmentation. Therefore, our proposed audio based occupancy inference model tackles a richer problem, where none of the speakers are discarded for handling the computational challenges. Crowd $++[6]$ proposed to combine pitch with MFCC to compute the number of people with an average error distance of 1.5 speakers. On the other hand our model improved the average error distance by a factor of two $(0.76$ Speakers).

Indoor Localization: UnLoc [14] proposed an unsupervised indoor localization approach exploiting environmental identifiable artifacts and specific signatures on single or multiple sensing dimensions using smartphones' different sensors readings (mainly from accelerometer, compass, gyroscope, and WiFi APs). [15] measured geomagnetic field in a way which is spatially varying but temporally stable, using an array of e-compasses to infer location. However they used a bunch of sensors or sensor arrays for location detection where as our model only used smartphones' magnetometer sensor to infer semantic location information of a gathering at zone/room level granularity. [16] used magnetic fingerprints with dynamic time-warping algorithm to predict location information with a $92 \%$ accuracy. Our model used standard Random Forest algorithm and achieved $98 \%$ accuracy to detect high level semantic location information of any gathering. IndoorAtlas location technology [17] utilized anomalies of ambient magnetic fields for indoor positioning. This platform provides the functionality for participatory sensing where the crowd can contribute by war driving magnetic signatures of an unexplored location.

\section{OVERALL SYSTEM ARCHITECTURE}

We envision developing a minimally invasive cost free robust mobile system for counting the number of people present at any time in any environment and enlighten their semantic location information. Our model boosts these capabilities by employing smartphones' magnetometer, microphone and accelerometer sensors. Our system as shown in Fig. 1, comprises of two subsystems, one deployed on smartphone and other in server. Using only acoustic sensing it is not always possible to predict the correct number of the occupants present in a specific location as some people get involved in a conversation while others remain silent. For example, in a class room scenario while professor lectures some of the students participate but majority of the students remain silent. Sensed data are stored in a data sink (sink) for posterior analysis in the mobile part of our proposed architecture consisting of accelerometer and magnetometer. In our model we propose to utilize microphone sensor based acoustic sensing in conjunction with accelerometer sensor based locomotive 
sensing for occupancy detection. For this joint collaborative sensing acoustic sensed data is being fed to the filter to collect Acoustic Fingerprint (AFP), consisting of content based audio. The AFPs being collected from all smartphones are sent to "Estimate Proximity" module residing on the server which helps distinguish the audio signals in vicinity and approximate the inclusion of a group of smartphones to form a single clique. Finally, "Optimum Node" module elects the clique leader (most informative smartphone) to record the audio data and notifies the condition of deactivation to the other smartphones from capturing the duplicate audio signal. It also helps in sorting the smartphone list based on their audio signal strength which is eventually utilized by locomotive "Signature Collection" module to opportunistically check-on and trigger the accelerometer sensor on the smartphones [18]. The server-side architecture consists of two main logical sub-components: i) Occupancy Context Model, and ii) Location Context Model. These models together form the inference engine of our proposed semantic location sensitive occupancy detection system.

\subsection{Occupancy Context Model}

It has two sub-modules, Acoustic Context Model and Locomotive Context Model.

\subsubsection{Acoustic Context Model (ACM)}

Our acoustic context model comprises of the following three modules.

Pre-processing: This module is the most trivial phase for acoustic signal processing. This module helps to perform the filtering and select the audio segment length dynamically. It finally helps remove all the noises, silences and produce smooth conversational data which is later passed to the feature extraction module.

Feature Extraction: This is the main basis for extracting all types of features which is utilized in the speaker estimation module. This module takes conversational samples and processes it through a series of data cleaning and feature extraction steps. It helps making frames from samples to calculate various features like MFCC, pitch etc. These features are later used by the speaker estimation module.

Speaker Estimation: This module serves as the core processor for occupancy counting. It takes MFCC as parameter and then measures the similarities between the audio frames and segments. Based on this similarity measures, it decides whether those speech segments are generated from distinct or same speaker. It keeps track of all the segments and their identities with respect to a specific person and finally helps count the total number of existing speakers during a conversational episode.

\subsubsection{Locomotive Context Model (LCM)}

It comprises of i) Signature Collection, ii) Feature Extraction, and iii) Occupancy Estimation modules. Signature collection module receives total number of people count from ACM module and the sorted smartphone list from the optimum module to opportunistically select a single smartphones' microphone sensor. Based on these two inputs, $L C M$ module makes decision on which smartphones' sensors are needed for further occupancy estimation. Feature extraction module calculates accelerometer sensor magnitude and feeds that into Occupancy Estimation module, which infers binary occupancy for each smartphone and finally helps counting the total number of people present in a conversational cum silent environment.

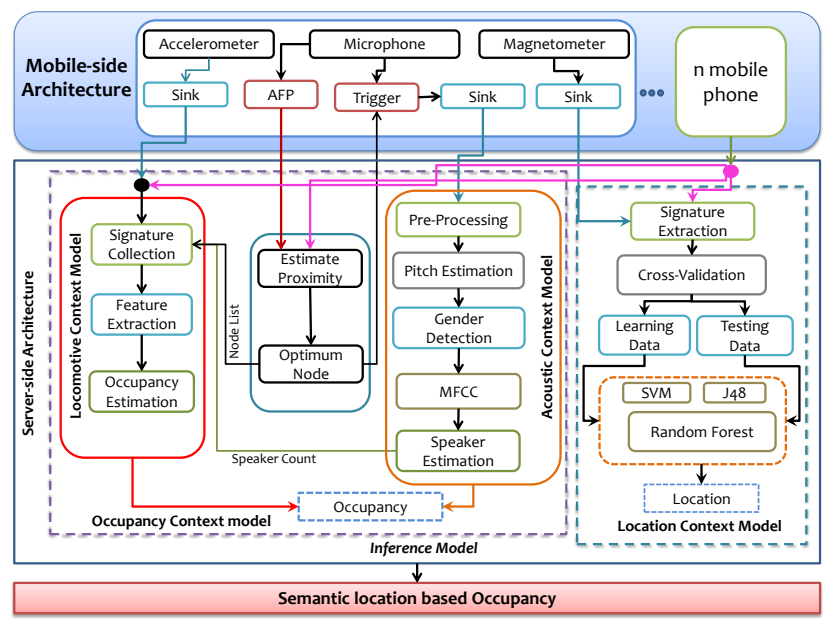

Figure 1: Architectural Overview of our Model

\subsection{Location Context Model}

Our Location Context Model consists of two sub-modules, i) Signature Extraction, and ii) Location Estimation. In signature extraction phase, we compute the feature vectors from smartphone's magnetometer sensor data. In the location estimation phase, we use that feature sets for crossvalidation to construct training and testing sets. After producing training and testing sets we apply machine learning techniques to infer location.

\section{DESIGN METHODOLOGY}

In this section we describe the details of our model design framework. We present an acoustic augmented locomotive sensing model for counting the number of people present in a conversing, non-conversing natural environment. We posit a magnetometer sensor based fingerprinting methodology to semantically localized the gathering.

\subsection{Occupancy Estimation Using Acoustic Sig- nature}

In this section, we describe occupancy estimation using our proposed acoustic sensing model. We look into the specific cases where all the occupants have been conversing. We first attempted to calculate the number of speakers engaged and consider three different phases to compute the number of personnel present. First We propose to create dynamic segments from the raw audio data and assume that each segment belongs to an individual person. We attempt to detect every speaker change point in the entire audio signal spectrum and assign one segment to one person to increase the counting performance of our occupancy detection algorithm. Speaker change point depicts the stopping point of one speaker and starting point of another speaker. Speaker change point detection algorithms have been investigated extensively [19][20][21], however, it is a complex process to detect speaker change point in conversational speech because utterance lengths can be extremely short, speaker changes may occur frequently, overlaps between the speakers may happen, and surrounding environment can be noisy. 
We first calculate confidence score for the entire audio segment which represents the probability of finding pitch within a segment. We then start finding confidence score from a small segment (32 ms) and increase the step size in the successive iterations and repeat this up to an audio segment of size 10 seconds. We calculated the variance of this confidence score and based on a lower variance associated with a specific segment we selected that segment length as one unit of conversation. If a segment has over $90 \%$ confidence, we considered it. As there are many audio segments with different segment lengths, we have chosen a segment length corresponding to a single person unit associated with a higher confidence score and greater number of audio segments with lower segment length. Fig. 2 shows various confidence scores for different segment lengths. We selected $2.72 \mathrm{sec}$ as segment length instead of $3.36 \mathrm{sec}$ when both have a confidence score of 1 , but first segment length admitted greater number of segments than the latter one. We have calculated this confidence score using YIN [22] algorithm by using nonoverlapping frames and skipped the best local estimate step. This help to determine on real time the unit audio segment which solely depends upon the recorded audio.

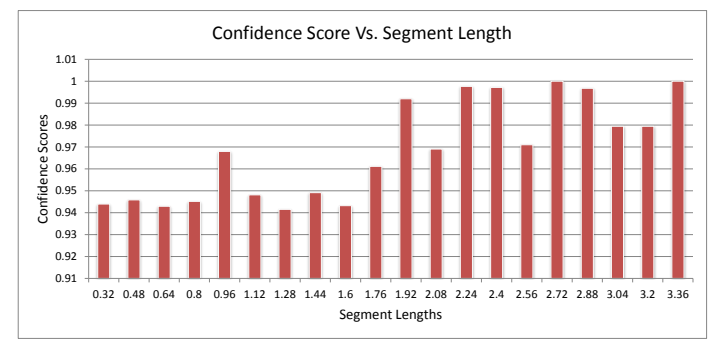

Figure 2: Confidence Scores for different segment lengths of a sample audio

As human voice ranges approximately $300 \mathrm{~Hz}$ to $4000 \mathrm{~Hz}$, we filter each of the segments based on that frequency range using band pass filter. After filtering the raw audio we have applied Hamming window to reduce the spectral leakage while creating audio segments. Consider a segment which contains $\mathrm{m}$ frames and each segment consists of frames $\left\{F_{1}\right.$, $\left.F_{2}, \ldots, F_{m}\right\}$. We calculated MFCC for each frame where each segment has corresponding MFCC feature vectors as $\left\{M_{1}, M_{2}, \ldots, M_{m}\right\}$. We also computed pitch for each segment to apprehend gender in the conversational data. Segment pitches are represented as $\left\{P_{1}, P_{2}, \ldots, P_{m}\right\}$, where the average pitch for male falls between 100 to $146 \mathrm{~Hz}$ whereas female pitch is within 188 to $221 \mathrm{~Hz}$, as demonstrated in [23]. Segments which fall within male frequency are marked as male and similarly for female. These two sets are then passed to our proposed people counting heuristic algorithm. Before passing these male and female segments for checking similarity measures, we calculated intra cosine angle of each segment to sort out both male and female segments. Next we have checked the similarity among inter-segments if it falls within our predefined threshold, $\theta_{t h}$ or not. If these segments have been similar, we have merged them to make a new segment and continued to check for the next segment with this newly created segment. If those segments have been dissimilar then we have moved forward and picked another segment to check similarity with the next one. The

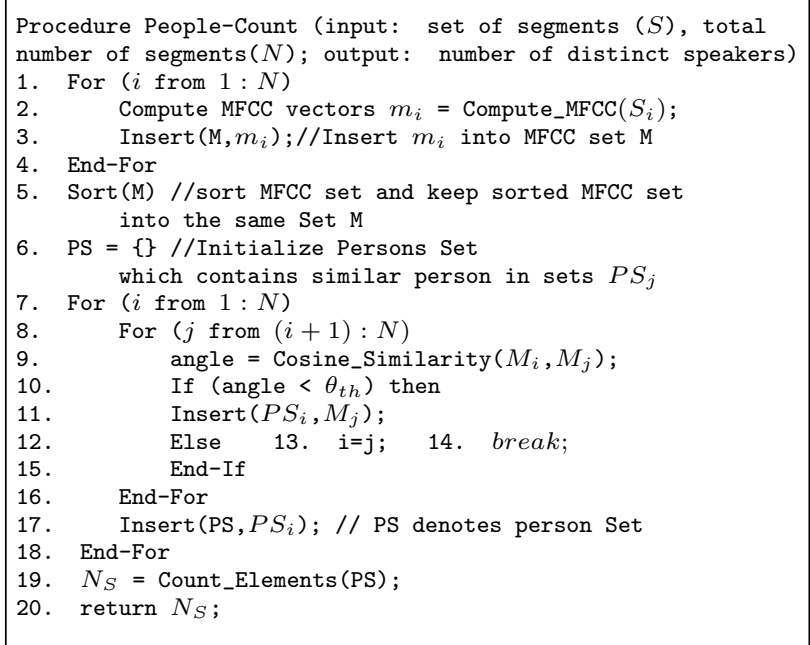

Figure 3: Acoustic People Count Algorithm

pseudo code of our proposed people counting heuristic has been shown in Fig. 3.

\subsection{Occupancy Estimation Using Accelerom- eter Signature}

In this section, we discuss our locomotive sensing model in absence of any conversational data or in a mixed environment where a group of people may talk and other listen silently. If a smartphone is stationary for a significant amount of time, on-board accelerometer sensor produces steady state signature which has no variation or spikes in terms of signal amplitude, whereas if there is a movement it generates a spike or corresponds to a steady-state signal alteration. To detect this abrupt changes in locomotive signal amplitude we propose to use change point detection based technique [24].

Change point detection helps to find the abrupt variation in the movement data stream. Our motivation in this work is to use change point to find the stray movements by finding abrupt changes in the accelerometer signals. These changes help inferring binary people counting (whether people are present or not). We investigated offline Baysian changepoint [24] detection based algorithm for inferring occupant's presence in $\mathcal{O}\left(n^{2}\right)$. Let the observed accelerometer data sequence be $x_{1: N}=\left\{x_{1}, x_{2}, x_{3}, \ldots, x_{N}\right\}$ where $N$ denotes the number of data points over time $T$. We partition this data sequence into non-overlapping region based on run length [25]. The length of each partition or time since the last change point occurred is defined as "run length". If there are $m$ partitions then the partition data set is denoted as $\left\{\rho_{1}, \rho_{2}, \rho_{3}, \ldots, \rho_{m}\right\}$. We also denote $x_{t_{i}: t_{j}}$ as the contiguous set of observations between time $t_{i}$ and $t_{j}$ inclusively. If the length of current run at time $m$ is denoted by $r_{m}$, then it can be defined as follows.

$$
r_{m}= \begin{cases}0 & \text { if change point occurs at }(m-1) \\ r_{m-1}+1 & \text { otherwise }\end{cases}
$$

Changepoints occur at discrete time points. The conditional probability that a change point occurs on time $t_{k}$ after the 
last change point at time $t_{k-1}$ is

$$
\begin{gathered}
\pi\left(t_{m} \mid t_{m-1}\right)=g\left(t_{m}-t_{m-1}\right), \quad \text { where } 0<m-1<n \\
\pi\left(t_{m}\right)=\sum_{j=0}^{m-1} g\left(t_{m}-t_{j}\right) \pi\left(t_{m-1}\right)
\end{gathered}
$$

where $\pi\left(t_{m}\right)$ is the prior probability of a change point at time $t_{m}$ and depends on the probability distribution of the observed data sequence and the preceding change point.

Changepoint detection algorithm computes predictive distribution $\pi\left(x_{n+1} \mid x_{n}\right)$ on a given run length $r_{m}$ taking the integration over the posterior distribution $\pi\left(r_{n} \mid x_{1: n}\right)$ which is computed using the following equation.

$$
\pi\left(r_{n} \mid x_{1: n}\right)=\frac{\pi\left(r_{n}, x 1: n\right)}{\pi\left(x_{1: n}\right)}
$$

It also finds out the joint distribution over the run length and the observed data as follows.

$$
\begin{array}{r}
\pi\left(r_{n}, x_{1: n}\right)=\sum_{r_{n-1}} \pi\left(r_{n}, r_{n-1}, x_{1: n}\right) \\
=\sum_{r_{n-1}} \pi\left(r_{n} \mid r_{n-1}\right) \pi\left(x_{n} \mid r_{n-1}, x_{1: n}\right) \pi\left(r_{n-1}, x_{1: n-1}\right)
\end{array}
$$

where $\pi\left(x_{n} \mid r_{n-1}, x_{1: n}\right)$ is the segment log likelihood which depends on the data $x_{n}^{(r)}$ and $\pi\left(r_{n} \mid r_{n-1}\right)$ is the change point probability which can be calculated as follows.

$$
\pi\left(r_{n} \mid r_{n-1}\right)= \begin{cases}H_{f}\left(r_{n-1}+1\right) & \text { if } r_{n}=0 \\ 1-H_{f}\left(r_{n-1}+1\right) & \text { if } r_{n}=r_{n-1}+1 \\ 0 & \text { otherwise }\end{cases}
$$

where hazard function $H_{f}(\eta)$ is calculated using $H_{f}(\eta)=$ $g(\eta) / \sum_{j=\eta}^{\inf } g(j)$. We employ this change point technique in our locomotive sensing model for designing binary occupancy detection algorithm. It has been built on the basis of the following three folds methodology. First, we calculate a-priori probability of two successive change points at a distance $d$ (run length). We use Gaussian based loglikelihood model [26] to compute log-likelihood of the data in a sequence $[s, d]$, where no change point has been detected. Second, we calculate log-likelihood for the entire signal $S[t, n], \log$-likelihood of data sequence $S_{s}[t, s]$ where no changepoint has been occurred between $t$ and $s$ and $\pi[i, t]$, the log-likelihood that the $i$-th changepoint occurs at time step $t$. Finally, We calculate the probability of a changepoint at time step $t$ by summing up the log-likelihoods for that sequence. Fig. 4 presents the changepoints and their probabilities which are being detected successfully in our proposed locomotive sensing model using smartphone's accelerometer sensor. We filter those changepoints based on empirically determined threshold probability $\left(\delta_{t h}\right)$ and infer presence of the occupants based on the admitted changepoint sequence. We also count the number of changepoints in the the data sequence which indicates movement score that represents how frequent a person moves. The overall algorithm has been summarized in Fig. 5 .

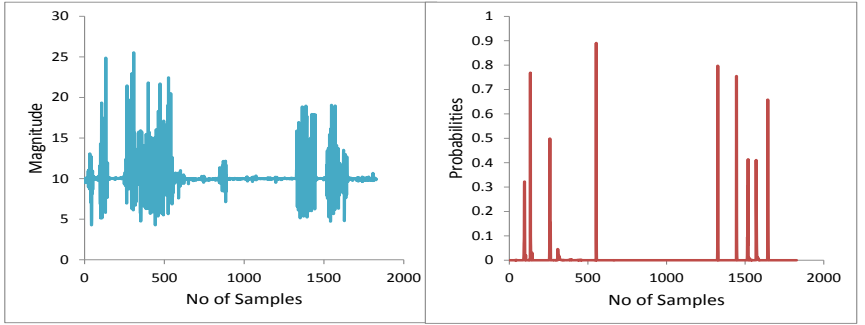

Figure 4: Magnitude of accelerometer signal (Left) and changepoints with probabilities of that signal (Right) due to a person's random movement patterns

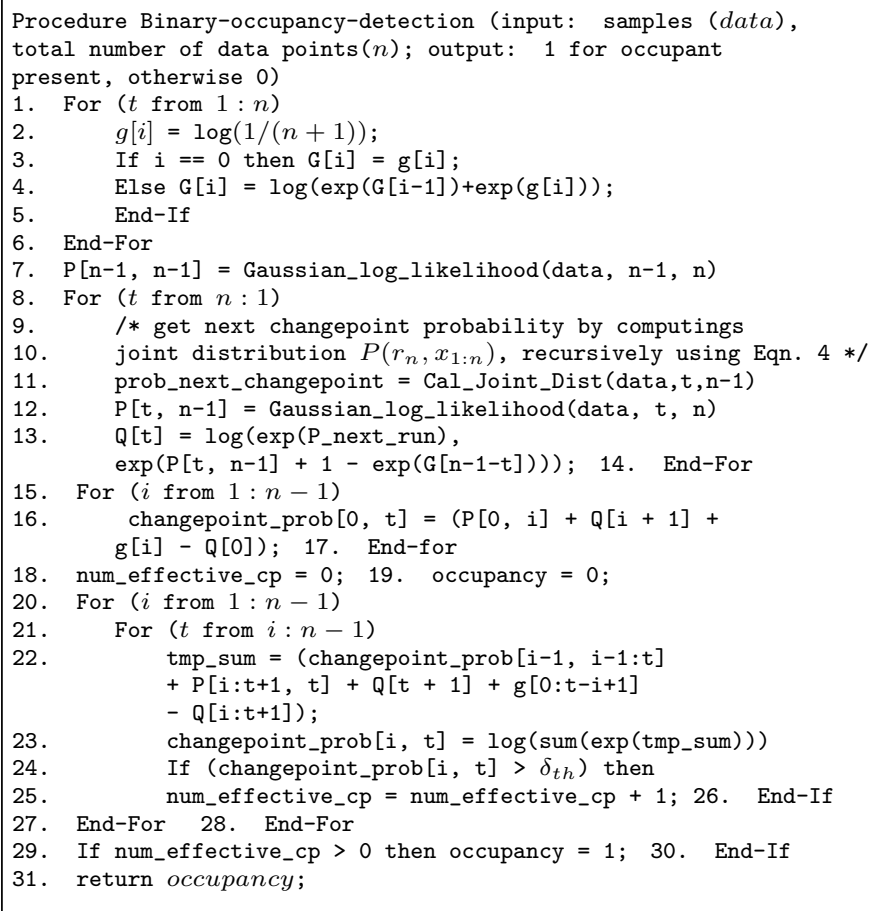

Figure 5: Binary Occupancy Detection Algorithm

\subsection{Location Estimation}

In this scenario our goal was to explore the possibility of inferring the location at zone/room level in different commercial and residential buildings by only using the smartphones' magnetometer sensor signals. Intuitively this is possible as different rooms have magnetic patterns that are distinct based on their unique structures and furniture layouts. This opens up the possibility that a sophisticated machine learning technique may learn to discriminate magnetic signatures belonging to different rooms. In our experiment, we collected magnetic signature of different rooms, office spaces and lobby area in an academic building using smartphones' magnetometer sensor. In a room, all furnitures and metallic objects generally remain fixed in positions and rarely are moved from one place to another. This gives us an intuition that each room has its own magnetic fingerprints which can be utilized to detect that specific room or semantic location.

We notice that magnetic sensor is sensitive to magnetic fluctuations in indoors specially near pillars and metallic objects. Fig. 6 represents this behavior where peaks occur 

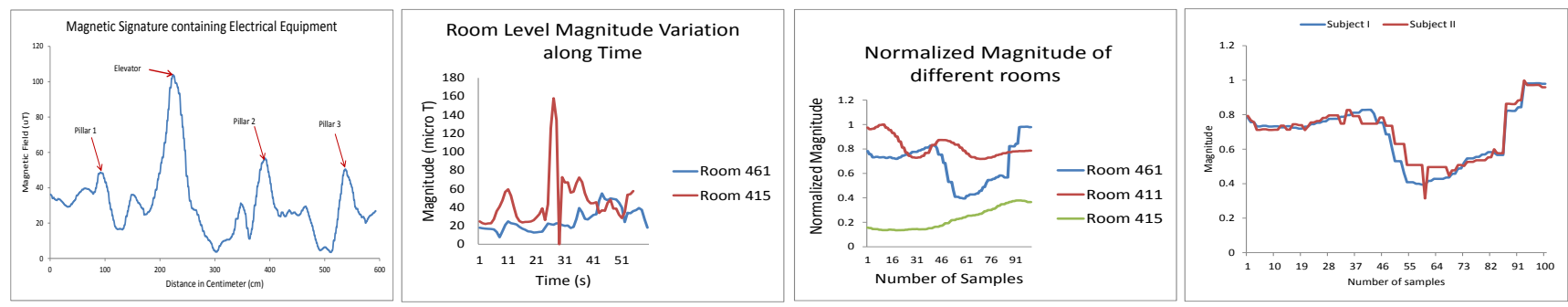

Figure 6: Magnetic signa- Figure 7: Magnetic signature Figure 8: Normalized magni- Figure 9: Normalized magniture variation for different equip- variation along time for two tude of magnetometer for differ- tude of room for different subments. rooms. ent rooms jects

near pillars, elevators etc., because pillars and elevators emit high magnetic fields. Magnetic fields produced by pillars are different for each floor because of their varying intensity level. This density characteristics guide with localization because each floor is independent in structure and height with other levels, from which it is also probable to infer floor level location. From this empirical observations, we conclude that each room has its unique magnetic fingerprint. We analyze different rooms data at University's Information Technology and Engineering (ITE) building for three months. Fig. 7 represents this analysis which depicts each room specific magnetic fingerprint helping to create a coarse localization model for pinpointing the semantic location of gatherings at zone/room level.

We also note that this magnetic signal differs not only for different indoor environment but also for phone's placement. This distraction has been optimized in two different ways - i) calibrating magnetic signals, and ii) calculating absolute magnitude. During our experimentation we observe that magnitude represents different fingerprints for separate indoor environment. Fig. 8 describes how normalized magnitude of different rooms varies upon total number of samples. Performing this experimentation over several rooms helps establish the fact that each room represents a different magnitude which may form their own fingerprint. We consider magnitude of magnetometer because for different persons with distinct movement, it does not deviate much other than little variations. Fig. 9 represents these characteristics where magnetic signature has been collected from two different people in the same room, both signals delineates same shape and almost same magnitude.

From this empirical study, we conclude that by only magnetic signature, it is difficult to estimate fine-grained indoor location in different indoor environments, for this reason we also consider mean, standard deviation and variance of different axes. Based on those feature vectors we generate two sets of data: training and testing using cross-validation process. We use training set to learn indoor characteristics by using different machine learning models and later use the testing set to predict location. To estimate fine-grained semantic location, we use SVM, J48, Random Forest classifiers.

\subsection{Crowdsourcing Magnetic Model}

We propose to use collaborative sensing or crowdsourcing to ease our ground truth data collection and location mapping process. We have divided the area of interest inside the ITE building as a grid of squared cells (details are provided in Section 5.2). We collected data from most fre- quently visited grids without any major obstruction. While crowdsourcing the unique characteristics of grid location, it was difficult to choose the right representation of data as analogous magnetic signatures of different grids in different locations were prevalent. As a result it was deemed necessary to display a potential set of locations from which the crowd would finalize the association of a semantic label with a particular observed magnetic signature pattern. Considering this we provide the floor information for a specific signature pattern, such that our crowdsourcing model will enable the crowd to choose the appropriate semantic location or room from that specific floor. Nevertheless the search space remains large as the possibilities of multiple rooms with similar magnetic footprints in a floor are quite abundance. We propose a simple grid mapping crowdsourcing model which reduces the search space by mapping the magnetic signature pattern of point of occupancy with the existing patterns and sorts the rooms according to the similarity measurement. Our model takes the Manhattan distance and the squared deviation of magnetic magnitude as input parameters for the mapped grids and search the repository of existing signature patterns database.

Consider a set of cell values found from a test pattern $X=x_{1}, x_{2}, x_{3} \ldots x_{n}$. First we take $x_{1}$ from $X$ and try to map this value with the cell values of existing patterns. We do not assume to have any prior idea regarding the organization of the cells in the test pattern. For mapping signature values we consider the deviation of \pm 2 which have been determined empirically according to our experiments. The patterns which matches the similarity value of a cell, we add them to our candidate set, $C$ and initialize a $n \times n$ distance matrix $\bar{M}^{(i)}$ and a $n \times 1$ deviation matrix $\overline{D^{(i)}}$ for each candidate $c_{i} . \quad \overline{M^{(i)}}$ records the manhattan distances_between the mapped cells in a candidate pattern $C_{i}$ and $\overline{D^{(i)}}$ stores the squared deviation between the mapped cell values. If we find similarities in multiple cell values in a single room signature pattern, we consider them as individual candidate. We take the next test pattern, $x_{2}$, in next iteration and do the similar operation like $x_{1}$, but this time we consider only the candidates in $C$. In this iteration, if the deviation and distance matrix of a candidate $c_{j}$ does not get updated then we discard it from the candidate set and reduce the search space. We recursively perform the same mapping for remaining grid values and compute the final matching candidate set $C_{F}$ with their corresponding distance and deviation matrices.

At this stage, it is still possible to have a large number of candidates in $C_{F}$. To tighten the search space, next we compute the error measurements for each candidate $E\left(c_{i}\right)$ and 
sort the candidates with respect to this value assuming that in an ideal conversational episode the participants remain in close proximity. We calculate $E\left(C_{i}\right)$ based on Eqn. 5 .

$$
\begin{array}{r}
E\left(c_{i}\right)=\sum_{p=1}^{m} X_{k, p}\left(\sum_{r=1}^{n}{\overline{M^{(i)}}}_{a, r} \overline{D^{(i)}}{ }_{r, b}\right)_{p, l} \\
\text { where } k=1, l=1,1 \leq a \leq n \text {, and } b=1
\end{array}
$$

After calculating the error measurements for each candidate, we sort $C_{F}$ and choose the first 10 candidates from $C_{F}$. We plot the magnetic signature pattern of these candidates and the test pattern. The crowd now have to choose the signature pattern in which they find the test pattern. In our experiments there were some cases where we observed empty candidate set. In these cases, we selected the last iteration's candidate set which was not empty. We also asked the crowd, if they found match with multiple candidates then they have to choose the earliest signature pattern.

\section{SYSTEM IMPLEMENTATION AND EVAL- UATION RESULTS}

We now discuss the detailed implementation and evaluation of our model framework.

\subsection{Tools and Resources}

We used Google Nexus-5 with built in microphone and three axes accelerometer sensor for our experiments. Our entire system comprises of two parts: i) sensing, and ii) classification and clustering, first one was implemented on Nexus5 and latter on the server. Application software was written in Java which utilizes Android Programming Interface (API) to sense microphone and accelerometer signals. Classification and clustering algorithms and our occupancy counting algorithm have been implemented on the server side using python.

\subsection{Data Collection}

Magnetic sensor signals are sensed through our android application and stored temporarily on mobile storage. We first collected magnetic data for training set, and subsequently for the testing set. We divided the room space into small regions each contains area $0.5 \times 0.5 \mathrm{~m}^{2}$ and named as cell. Thus each room forms grid containing cells. We collected data from each cell for 5 minutes both clockwise and counter clockwise direction to form the training set. We also maintain fixed height (approximately 4 feet from the floor) when collecting our ferromagnetic fingerprint because it also depends on the height. Partial 3rd floor map is shown in Fig. 10. It shows sample data collection path of room number 305 where green line shows how the grid forms and red line shows the data collecting path in both direction along the grid. We use sampling rate $5 \mathrm{~Hz}$ for magnetometer sensor data. We implemented the acoustic sensing and collected conversational data from different places at different times in natural settings. Conversational data have been collected and properly anonymized during the spontaneous lab conversation among the students (without making the occupants aware of it), lab meeting, and general discussions in the lobby/corridor in presence of a variety of surrounding noise levels. The demographic for our conversational data collection was 1-10 persons (with 5 females and 5 males) in age group of 18-50 years. The acoustic data were collected at a mono sampling rate of $16 \mathrm{kHz}$ at $16 \mathrm{bit}$ pulse-code modulation (PCM).

\subsection{Privacy}

One of the major concerns of smartphone based acoustic signal processing is privacy. This concern becomes more serious when smart-phone records the conversation data. Our counting algorithm determines the number of speakers in this environment in an anonymized manner. We used text file as cover in which our recorded audio is embedded. A secret key is induced for embedding and extraction process which is known by both the sender and the recipient. A steganographic function takes cover file as argument and then embeds audio file and key to produce stego as output which is sent to our server. A reverse steganographic function on our server side takes stego file and key as parameter and produces audio file as output. There are different steganographic methods (i.e. LSB coding, parity coding, phase coding) but we used the simplest method, least significant bit algorithm which replaces the least significant bits of some bytes in the cover file to hide a sequence of bytes containing hidden data. To generate the stego file, the algorithm first converts each character of the cover file into bit stream followed by converting the audio file into bit streams and finally replacing LSB bit of the cover file with the bit of the audio in the secret information. We also ensured that the size of the file was not changed during this encoding and it was suitable for any type of audio file formats.

\subsection{Magnetic, Acoustic and Locomotive Fea- ture Extraction}

We discuss different features relevant to our acoustic, locomotive sensing and localization technique in this section.

Magnetic Features: For location detection we used only magnetometer sensor. Smartphones' magnetic sensor provide three axes values $\mathrm{x}, \mathrm{y}$ and $\mathrm{z}$ axis. From these values we calculated magnitude using $m=\sqrt{x^{2}+y^{2}+z^{2}}$. We considered only the resultant magnitude to mitigate variations of the readings resulting from smartphone's different axes based on different positions. We also calculated mean, variance, and standard deviation of each readings and combined those features to generate the feature vectors.

Acoustic Features: We generated two basic features which are used in the speaker identification - MFCC and Pitch. Each feature has been described in details in the following. i) $M F C C$ is one of the most significant features which is used for acoustic processing. We followed the following steps to process it. 1. Take the Fourier transform of (a windowed excerpt of) a signal, 2. Map the powers of the spectrum obtained above onto the Mel scale using triangular overlapping windows, 3 . Take the logs of the powers at each of the Mel frequencies, 4. Finally, take the discrete cosine transform of the list of Mel log powers. We excluded the first co-efficient of MFCC and then chose 20 coefficients as feature vectors. ii) Pitch is defined as the lowest frequency of a periodic waveform. It is the discriminative feature between man and woman. Human voice pitch interval falls within the range of $50 \mathrm{~Hz}$ to $450 \mathrm{~Hz}$ [23]. We calculated pitch of different segments using YIN [22] algorithm. We used 32 msec hamming window with $50 \%$ overlap for computing the Pitch and MFCC feature.

Locomotive Features: We considered the magnitude of the accelerometer data as our locomotive feature in order to 

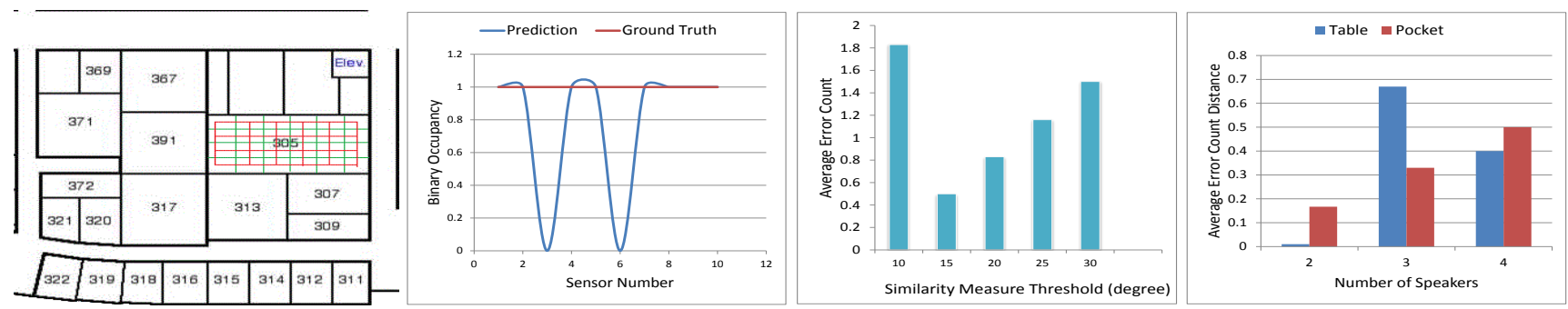

Figure 10: Sample Magnetic data collection path
Locomotive Figure 12: Performance with Sensing-based Occupancy Count different cosine measures
Figure 13: Occupancy count
over different phone positions mitigate calibration.

\subsection{Accuracy Metrics Definition}

To evaluate and compare the performance of our location sensitive occupancy model, we first define the following metrics. i) Occupancy Metric: We computed the average error count as the normalized predicted occupancy metric represented by $\frac{|E C-A C|}{N}$, where EC, AC, N respectively denote the estimated people count, actual people count and number of samples respectively. We presented only the absolute value in order to avoid any positive or negative contribution. ii) Location Metric: For evaluating location measurement we consider the following metrics. Average Precision $\left(\frac{T P}{T P+F P}\right)$, Average Recall $\frac{T P}{T P+F N}$, Average F-1 Score $\left(\frac{2 \times \text { Precision } \times \text { Recall }}{\text { Precision }+ \text { Recall }}\right)$, where TP, FP, TN and FN are the number of instances of true positive, false positive, true negative and false negative respectively. iii) Location Prediction Error: It is defined as the mean absolute error between predicted and actual value of the estimated variable. This error is expressed as Mean Absolute Error $=\frac{1}{n} \sum_{i=1}^{n}$ | $\left(f_{i}-y_{i}\right) \mid$, where $f_{i}$ is the prediction and $y_{i}$ is the actual value.

\subsection{Occupancy Counting Results}

We evaluated our opportunistic occupancy counting algorithm in four scenarios. i) No conversation among occupants, ii) All occupants are conversing in a single clique, iii) Occupants are conversing in multiple cliques, and iv) Mixed conversing and non-conversing occupants.

For the first scenario, when no occupants are involved in a conversation we used the accelerometer to count the occupancy. Each accelerometer sensor provides binary occupancy indication based on our change point detection algorithm as discussed in section 4.2 which computes the total number of people present in the environment. Fig. 11 shows the total number of people successfully counted using our locomotive sensing model. We note that our locomotive sensing model achieves $80 \%$ accuracy ( 8 out of 10 people) in predicting occupancy when most of the users carry their smartphones with them.

Our opportunistic sensing system plays a critical role when all occupants have been conversing in a single clique. Our system helps to activate a single microphone for occupancy counting and deactivate all other microphones and accelerometer sensors based on the server's feedback (details are omitted due to space constraints). Fig. 12 depicts the effect of cosine distant similarity measures on our occupancy counting algorithm as shown in Fig 3. We noticed that similarity distance angle measures (in degree) play a pivotal role on reducing the error count of occupancy inference. In our experiments with 3 people conversing, we found that 15 degree similarity measure threshold is an appropriate choice for consideration to reduce the error count for our proposed adaptive people counting algorithm.

We also have run experiments in an uncontrolled environment (completely in a natural setting) without imposing any restrictions on smartphones relative positions and distances from each other or from the server. Fig. 13 reports the average error count distance $\approx 0.5$ with respect to different positions of the phone. It is noted that when smartphone is placed on the table and two persons speak the error count becomes zero, but when three persons start speaking, error count tends to become slightly higher due to the ambient noise and overlapped conversation.

Fig. 14 depicts that error count increases as single clique leader's distance from other occupants increases. We note that for a 3 meter distance error count becomes close to two which confirms that even for a large internal distance separation among the conversing occupants our acoustic sensing model performs quite well.

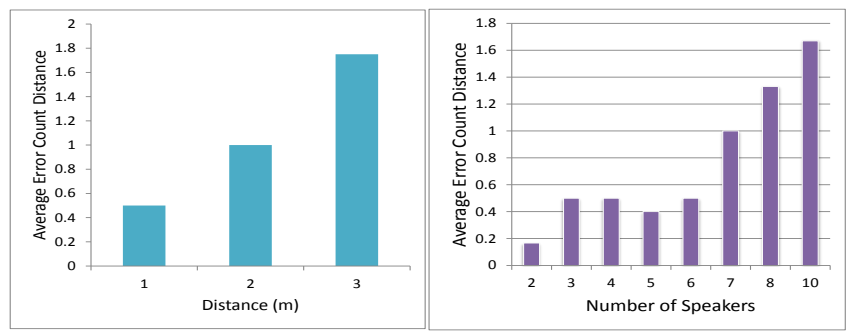

Figure 14: People counting Figure 15: Accuracy vs. vs. phone distance

Number of People

Fig. 15 presents the performance of our people counting algorithm where users speak naturally with overlapped conversations. It is observed that average error count is 0.1 for 2 people and 1.7 for 10 people when conversing together. Thus the overall average error count is 0.76 with number of users present varying from 2 to 10 establishes that our acoustic-based occupancy counting algorithm performs well even in a crowded environment.

In our third scenario, where occupants are conversing in multiple cliques (three cliques in our experiment) we deployed three microphones and accelerometer sensors which are chosen based on the proximity measure from the server to infer the occupancy. Fig. 16 shows the intra-group count in presence of conversational data with distinct clique formation. In our experiments, first group has 5 occupants (2 men and 3 women), second group has 6 occupants (3 men 

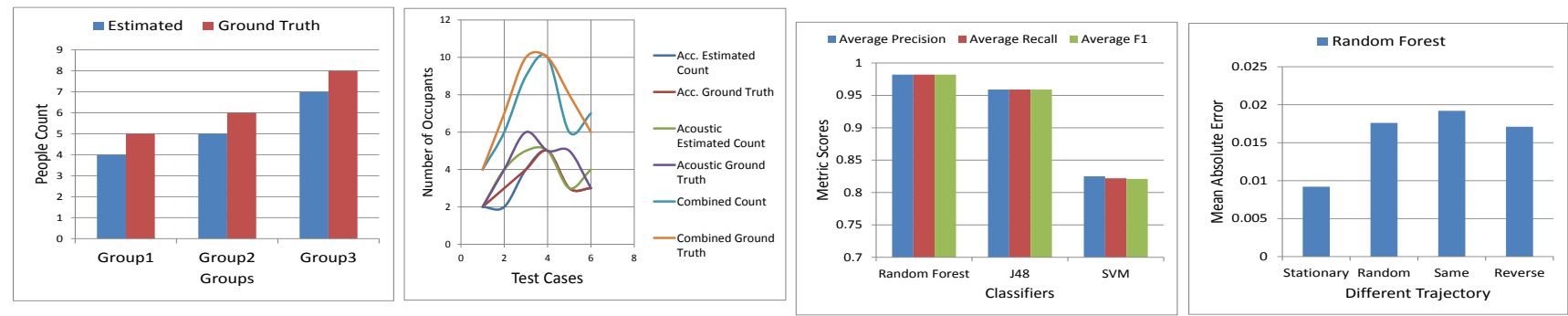

Figure 16: People Counting Figure 17: Locomotive Aug vs. Multiple Co-located Group mented Acoustic Occupancy of Speakers

Count Figure 18: Location estimation
errors for different classifiers
Figure 19: Location estimation error vs different trajectories and 3 women) and last group has 8 occupants ( 4 men and 4 women). We observe that the mean error count is $\approx 1$ for even our group based acoustic sensing model which attests the promise of our occupancy detection model in different real life scenarios.

\begin{tabular}{|l|l|l|}
\hline $\begin{array}{l}\text { Number of } \\
\text { Speakers }\end{array}$ & $\begin{array}{l}\text { Crowd++ } \\
\text { (Error } \\
\text { Count) }\end{array}$ & $\begin{array}{l}\text { Our } \\
\text { model } \\
\text { (Error } \\
\text { Count) }\end{array}$ \\
\hline 2 & 0.5 & 0.167 \\
\hline 4 & 2.33 & 0.5 \\
\hline 6 & 2.5 & 0.83 \\
\hline Average & 1.78 & 0.5 \\
\hline
\end{tabular}

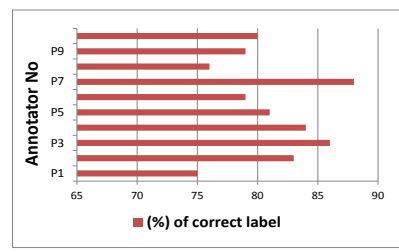

Table 1: Comparison (Average Error Count) between Figure 20: Results of our Crowd ++ and Our model Magnetic Crowdsourcing model

In our last scenario, where some people speak and some people remain silent arise, we propose to utilize our hybrid locomotive cum acoustic sensing model to infer total number of occupants. For example, consider a scenario where six persons are involved in conversation while four remain silent. For conversing population, we activate either a single microphone sensor if there is a single clique or multiple microphone sensors if there are multiple conversing cliques as determined by our "Estimate Proximity" module implemented on the server. We use mean error count estimation to infer the number of people conversing. To estimate the number of people who are not involved in that conversation, we utilize our locomotive sensing model which postulates binary occupancy using change point detection applied on the accelerometer's signal and finally infers the total number of silent people. Fig 17 plots overall occupancy counting performance based on our hybrid approach. For example, when there are ten people and 6 persons converse in a single clique and 4 persons remain silent, our acoustic sensing estimates 5 people out of 6 and locomotive sensing estimates 4 people out of 4 , resulting in total of predicting 9 people out of 10 . We have compared the performance of our model with Crowd ++ framework [6] for counting the number of people. Table 1 shows that the average error count distance for Crowd ++ is 1.78 where as for our model it is 0.5 , more than a three fold increase in accuracy for inferring the total number of people.

\subsection{Location Estimation Results}

Fig. 18 presents the location estimation error of an occupancy gathering using different classifiers. The Random Forest classifiers perform best with an average precision, recall and F1 score of 0.98 .
We also validated our location model through different test cases where we consider $i$ ) different trajectories, ii) different times of a day, and iii) different rooms with varying number of occupants.

We conducted our experiments following different trajectories, like keeping mobile phone on the table, following the same or reverse directions when collecting data and finally, collecting data randomly for a room. We noted that these different movement patterns do not affect much in the performance of our occupancy gathering location determination model. Fig 19 shows errors for different movement patterns. We find that stationary pattern shows better accuracy while moving in the same direction gives higher error rate. Average errors are close to 0.015 , which is quite acceptable with a minor number of false positives or true negatives.

Fig. 21 depicts the varying nature of the magnetic signature during the different times of a day. We observe that the location estimation of any gatherings is similar during the different times of a typical day. It shows error ranges approximately from 0.015 to 0.03 due to the global variation of weather and other magnetic factors making our model as time invariant.
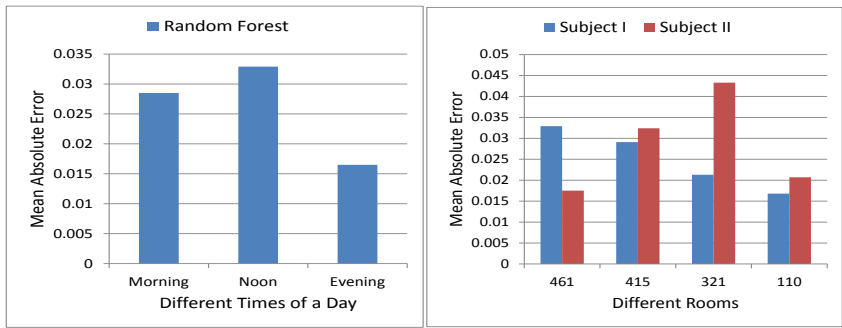

Figure 21: Location estimator Figure 22: Location estimaerror during the different time tion error in different rooms of a typical day with different occupancy size

We also ran experiments for location sensing model with respect to different rooms at different floors in ITE building with a different set and size of the occupants. From Fig. 22, we do observe that the mean absolute error approximately varies in the range of 0.015 to 0.04 which has negligible effect on the performance of our location sensitive occupancy determination model. We observed some discrepancies between different subjects data for room 321 and room 461. After investigating we found that the discrepancies happened due to unusual magnetic inferences of electronic devices present while collecting data for subject II. To evaluate our crowdsourcing model, we ran a simulation of our magnetic crowdsourcing model in Vowpal Wabbit(VW) 
toolkit [27]. We implemented our mapping algorithm on the server side and then used the function active_interactor of VW to interact with the users. We showed 10 magnetic signature patterns and 1 test pattern to an user and asked him to choose the magnetic signature pattern in which he/she finds the test pattern. 10 participants participated in the crowdsourcing task and in Fig 20 we show the overall accuracy for each participants when given 15 pattern matching tasks. Average accuracy of gaining correct annotation for these 15 patterns is $\approx 81 \%$ which is adequately high. Our results indicate that the probability for getting noisy labels is very low and the crowd annotated data can be chosen as input to the classifier.

\section{DISCUSSION AND FUTURE WORK}

In the current version of our work, we have assumed that people keep their smartphone in the pocket or in the hand which might not be ideal in some cases. In future our plan is to make our architecture more robust and independent of smartphones' location. The performance of our counting algorithm does not get affected by TV or radio sounds as TV or radio follows different modulation techniques which make it easier for us to remove those external noises from resultant audio signal systems. We have used source separation where significant overlap between human conversation and TV occurs. In the current implementation, location mapping process is independent of the classification process. In future we plan to develop and integrate a combined mapping and classification model. We also plan to investigate fine-grained floor level location using smartphone barometric sensing. We plan to investigate more advanced opportunistic sensing model considering microphone, accelerometer and magnetometer sensor participation not only based on a serverbased architecture but also based on an inter-smartphone distributed collaborative sensing based approach.

\section{CONCLUSIONS}

In this paper, we presented an innovative system to infer the number of people present in a specific semantic location which opportunistically exploit accelerometer and microphone sensor of smartphone for people counting. We proposed an acoustic sensing based unsupervised clustering algorithm by addressing the underpinning challenges evolving from naturalistic overlapped and sequential conversation to infer the occupancy in an environment. We posit a change point detection based locomotive sensing model to infer the number of people in absence of any conversational episode. We implement an opportunistic context-aware client-server based architecture to leverage smartphones' microphone, accelerometer and magnetometer sensors and combine our acoustic sensing with locomotive and semantic location sensing model to better predict the location augmented occupancy information. We have also demonstrated a novel crowdsourcing model for reducing the effort of collecting location information at zone/room level at large scale. Our experimental results hold promises in a variety of natural settings with an average error count distance of 0.76 in presence of 10 users. We believe this investigation holds promises and helps to open up many new research directions in this opportunistic multi-modal sensing domain.

\section{Acknowledgement}

This work is supported partially by the NSF Award \#1344990, and Constellation $E^{2}$ : Energy to Educate Grant.

\section{REFERENCES}

[1] Lawrence Rabiner and Biing-Hwang Juang. Fundamentals of Speech Recognition. 1993.

[2] Tanzeem Choudhury and Alex Pentland. Sensing and modeling human networks using the sociometer.

[3] D.B. Jayagopi and et al. Modeling dominance in group conversations using nonverbal activity cues. Proc. of IEEE TASLP (2009).

[4] Hong Lu, A. J. Bernheim Brush, and et al. Speakersense: Energy efficient unobtrusive speaker identification on mobile phones. In Proc. of PerCom (2011).

[5] Rijurekha Sen, Youngki Lee, and et al. Grumon: Fast and accurate group monitoring for heterogeneous urban spaces. In Proc. of SenSys (2014).

[6] Chenren Xu, Sugang Li, and et al. Crowd++: Unsupervised speaker count with smartphones. In Proc. of UbiComp (2013).

[7] Youngki Lee, Chulhong Min, and et al. Sociophone: Everyday face-to-face interaction monitoring platform using multi-phone sensor fusion. In Proc. of MobiSys (2013).

[8] Robert Tomastik and et al. Model-based real-time estimation ofÂăbuilding occupancy during emergency egress. In Proc. of PED (2008).

[9] Ebenezer Hailemariam and et al. Real-time occupancy detection using decision trees with multiple sensor types. In In Proc. of SimAUD (2011).

[10] Hong Lu, Wei Pan, and et al. Soundsense: Scalable sound sensing for people-centric applications on mobile phones. In Proc. of MobiSys (2009).

[11] R.E. Yantorno B.Y. Smolenski U.O. Ofoegbu, A.N. Iyer. A speaker count system for telephone conversations. In Proc. of ISPACS (2006).

[12] A. N. Iyer, U. O. Ofoegbu, R. E. Yantorno, and B. Y. Smolenski. Blind Speaker Clustering. In Proc. of IEEE ISPACS (2006).

[13] Alessio Agneessens, Igor Bisio, and et al. Speaker count application for smartphone platforms. In Proc. of IEEE $I S W P C$ (2010).

[14] He Wang, Souvik Sen, and et al. No need to war-drive: Unsupervised indoor localization. In Proc. of MobiSys, (2012).

[15] Jaewoo Chung, Matt Donahoe, Chris Schmandt, Ig-Jae Kim, Pedram Razavai, and Micaela Wiseman. Indoor location sensing using geo-magnetism. In Proc. of MobiSys (2011)

[16] K.P. Subbu, B. Gozick, and R. Dantu. Indoor localization through dynamic time warping. In Proc. of SMC (2011).

[17] Indooratlas. https://www. indooratlas.com/.

[18] Luis A Castro, Jesús Favela, and et al. Collaborative opportunistic sensing with mobile phones. In Proc. of UbiComp (2014).

[19] Jitendra Ajmera, I McCowan, and H. Bourlard. Robust speaker change detection. Proc. of IEEE Signal Processing Letters (2004).

[20] Daben Liu and Francis Kubala. Fast speaker change detection for broadcast news transcription and indexing. In Proc. of EuroSpeech (2009).

[21] Lie Lu and Hong-Jiang Zhang. Real-time unsupervised speaker change detection. In Proc. of IEEE Pattern Recognition (2002).

[22] Alain de Cheveignéb and Hideki Kawahara. Yin, a fundamental frequency estimator for speech and music. J. Acoust. Soc. Am (2002).

[23] Ronald J Baken and Robert F Orlikoff. Clinical measurement of speech and voice. Cengage Learning (2000).

[24] Paul Fearnhead. Exact and efficient bayesian inference for multiple changepoint problems. In Proc. of Statistics and computing (2006).

[25] Xiang Xuan and Kevin Murphy. Modeling changing dependency structure in multivariate time series. In Proc. of ICML (2007).

[26] Kevin Murphy Xuan Xiang. Modeling changing dependency structure in multivariate time series. In Proc. of ICML (2007).

[27] Vowpal wabbit. http://hunch.net/ vw/. 